\title{
Review of Transmission Fixed Costs Allocation Methods
}

\author{
Zhaoxia Jing, Xianzhong Duan, Member, IEEĖ, Fushuan Wen, Yixin Ni, Senior Member, IEEE, and \\ Felix F. Wu, Fellow, IEEE
}

\begin{abstract}
In the context of competitive electricity markets, transmission fixed costs should be fairly allocated to transmission users. A reasonable allocation method could lead to efficient utilizations of existing transmission facilities and, at the same time, provide economic signals for guiding future generation planning and load sitting. In this paper, a comprehensive literature survey is made on available methods of transmission fixed cost allocations. The review is conducted from several different aspects including: costs to be allocated, entities to pay the costs, system states to be based on, cost allocations of unused capacities, pricing of counter flow and that of reactive power, and allocation principles and methods. In addition, the characteristics of each method are analyzed and compared with those of the others.
\end{abstract}

Index Terms-- electricity market, transmission pricing, fixed costs allocation.

\section{INTRODUCTION}

$\mathrm{M}$ ANY countries in the world are carrying on or will carry on the electricity industry reform. Reforms are always full of uncertainties and may bring enormous disaster instead of economic benefit in some cases. To a great extent, the success or failure of the electricity industry reform depends on the design of the market rule.

An important aspect in the electricity market design is the transmission pricing method. One of the preconditions of establishing competitive electricity marketplace is TOA (transmission open access), which will provide the fair market for generation competition, and assure the transmission facilities to be fairly and un-discriminatorily used by the transactions. In order to achieve TOS, the reasonable transmission pricing method is very important. What's more, it has significant effect on the security and efficient operation of power systems.

According to microeconomics theory, when the price of a commodity or service equals to its marginal cost, the greatest social welfare can be achieved. But for industries existing

This project is jointly supported by National Key Basic Research Special Fund of China (No. G1998020305), a seed funding project from the University of Hong Kong, and a specialized research fund for the doctoral program of higher education (SRFDP), China.

Z. X. Jing and X. Z. Duan are with the School of Electrical and Electronics Engineering, Huazhong University of Science \& Technology. Wuhan 430074 China (e-mail: jingzhaoxia (d) hounail.com xzduanz (ôn 263.net).

F. S. Wen, Y. X. Ni and F.F. Wu are with the Department of Electrical and Electronic Engineering. The University of Hong Kong, Pokfulam Road, Hong Kong (e-mail: lswen@eec.hku.hk, yxni@etc.hku.hk, ffwu@eec.hku.hk). economies of scale, since the average cost is lower than the marginal cost, the producer will achieve negative profit if the commodity or service is priced with marginal cost method. The widely used two-parts tariff includes two parts, i.e. the usage tariff and the fixed tariff. The usage tariff is usually priced with Short-Run Marginal Cost (SRMC) method based on the actually usage of the customers and it can recover all the variable costs and a small part of the fixed costs. The fixed tariff usually has no relationship to the actual usage and is used to recover most of the fixed costs. The two-part tariff method can make the supplier to recover all the costs and provide certain economic signal. If the fixed tariff can be decided reasonably, this method can lead to the best allocation of the economic resources.

Significant economies of scale exist in the transmission sector and transmission services are often priced with twopart tariff method. The usage tariff of transmission service is also called energy tariff and the fixed tariff is also called demand tariff. The energy tariff can be decided with the spot pricing method [1], in which the price of transmitting power between two nodes is the difference of spot prices of the two nodes. As to the calculation of the demand tariff, it becomes a difficult problem due to some characteristics of the transmission network.

The energy tariff, which includes transmission losses and congestion costs, can be used to recover all the transmission variable costs and a small part of the transmission fixed costs. The demand tariff is used to allocate the residual fixed costs among the transmission users. When the marginal cost based method is used for pricing, the costs recovered by demand tariff is also called complementary costs or supplementary costs. With the marginal cost based pricing method, the ratio of transmission fixed costs recovered through the demand tariff is related to the conditions of the actual systems. For example, the ratio is $15 \%$ in Chile but is less than $4 \%$ in Bolivia [2].

There are two kinds of transmission facilities, that is, the Connection Facilities used to connect the network users (generators or loads) to the transmission network and the Network Facilities used to transport the electricity to other places of the network. This paper mainly discusses the allocation methods of the transmission fixed cost related to the Network Facilities among the transmission users. In the following sections of this paper, the term "transmission cost" or "cost" all refer to this part of the transmission costs and the 
term "user" refers to transmission user.

Many allocation methods have been proposed, and some of them have been used in the actual electricity market. But at present, none of them is widely recognized. This paper makes overall, systematic summary and analysis of the existing allocation methods which are classified according to seven different criteria: costs to be allocated, entities to pay the costs, system state to be based on, cost allocation of unused capacities, pricing of the counter flow and that of reactive power, and allocation principles and methods.

\section{COSTS TO BE ALLOCATED}

According to the costs to be allocated, the allocation methods can be classified into three groups: rolled-in cost method, incremental cost method and composite cost method [3].

In rolled-in pricing method, all existing transmission system costs (embedded costs) and the new costs of system expansion, regardless of their cause, are firstly summed up ("rolled in") into a single number and this cost is then allocated among various users of the transmission system, including the utility native customers and wheeling transactions. The wheeling transaction is defined as the transmission of electric power for other entity(ies) by a utility that neither generates nor intends to use the power as a system resource for meeting its own native load; and the native load for a utility is defined as all power sales by the utility, both inside and outside utility's service territory.

In incremental cost pricing method, only the new transmission costs caused by wheeling transactions, that is, the incremental cost, will be considered to evaluate transmission charges for wheeling transactions. The existing system costs will be the responsibility of utilitics' present customers.

In composite embedded/incremental pricing method, both the existing system costs and the incremental costs are considered in evaluating overall transmission charges. The embedded costs are allocated among all the transmission users, including the native customers and the wheeling transactions, while the incremental costs are allocated among wheeling transactions. The total cost allocated to a wheeling transaction is the sum of the allocated embedded cost and incremental cost. An exception in implementing this method is the "or" pricing method proposed by FERC of the United States [3], in which the price of a wheeling transaction is based on the higher of the embedded cost and the incremental cost of the transaction.

The main difference among the three methods lies in the ways dealing with the wheeling transactions. In the rolled-in cost pricing method, all transmission users, including the native customers and wheeling customers, are treated equally; while in the incremental cost pricing, the native customers are regarded as the present customers and should pay for the existing system costs, while the wheeling customers are regarded as new customers and should thus pay for the incremental costs. The composite pricing is the integration of the first two methods.

When considering furthermore, the different methods reflect different cost allocation target: the rolled-in method emphasizes on the economic efficient while the incremental cost method much emphasizes on the fair principle [4].

\section{ENTITIES TO PAY THE COSTS}

According to the entities to pay the costs, the allocation methods can be classified into bilateral transaction based (BTB)method and node based (NB) method.

BTB method allocates the costs to bilateral or/and multilateral transactions according to a certain principle [513]. They are mainly used in bilateral/multilateral typed markets. If a Trade Hub is defined and all market participants in the power pool are regarded to trade with the Trade Hub, BTB methods can also be used in Pool typed markets. Most electricity markets in the United States use BTB methods.

$\mathrm{NB}$ method allocates the costs to generators, loads or both based on node injections, the commercial transactions not being considered [2, 14-19]. How to allocate the total costs between generators and loads is an arbitrary decision. From the viewpoint of promoting the long-run investment of generators and loads, the costs should be allocated to both generators and loads according to their usage of the network. Since the location of a generator is more sensitive to that of a load, the costs allocated to generators should be larger than those allocated to loads [20]. From another point of view, that is, to minimize the distortion of the demand charge to the economic signal provided by the SRMC, according to the concept of Ramsey pricing, more costs should be allocated to loads [17]. Most electricity markets in Latin America countries use NB methods, such as Argentina, Bolivia, Chile, Colombia and Peri [2].

BTB method emphasizes on commercial arrangement, while NB method emphasizes on physical connection and usage. Since bilateral or multilateral transaction is a commercial concept, it does not mean the actual power flow. For example, a transaction from A to B does not mean that all the generation from $\mathrm{A}$ flow to $\mathrm{B}$, or the power consumed by $\mathrm{B}$ all come from A. In fact, as it is impossible to dye the electrons and check the color, there is not a meaningful measurement scheme or a theoretically based evaluation method to trace the power flow in transmission networks. From this point of view, BTB method is not reasonable. But the node prices in NB methods are easily affected by the choice of reference node while the charges for bilateral or multilateral transactions in BTB methods are not so easy affected by the choice of reference node.

In some cases, for example, for the bilateral transactions, when evaluating the transmission usage by DC distribution factors, the costs allocated to a transaction using the BTB method will equals to the total costs allocated to the corresponding nodes using the NB method. 


\section{SYSTEM STATES TO BE BASED ON}

Under different system conditions, the transmission usage, the influence on the network and the benefit from the services of the transmission users will all be different. One or more system states in the pricing period (usually a month or a year) must be selected as base states to be based on.

\section{A. System peak state}

In this method, only system peak state, or coincidence peak state, is considered. The costs are allocated among users according to their transmission usage or benefit at the time of system peak flow $[14,15,21,22]$. If the quantity of the power transmitting of a user is zero at the time of system peak flow, this user will not need to pay for any costs.

\section{B. Weighted multi-states}

In this method, the transmission usage and benefit of the user at more than one system states are studied [2]. For example, consider 6 system states: weekday and weekend in summer, winter and autumn.

\section{User peak state}

In this method, the costs are allocated in proportion to the maximum power flow of each user. In [6], for transactions that the quantities of transacted power are variable, the transmission usage of each transaction is cvaluated by the maximum flow that the transaction may cause in all system conditions. This method is also called non-coincidence method.

\section{Maximum line flow}

In these methods, for the cost of each branch, the system state, based on which the cost is allocated, is that when the flow over the branch is the maximum flow [23]; that is to say, the costs of different branches are allocated according to different system states.

\section{E. Game theory based}

Considering the fact that the sum of maximum flow caused by all users is large than the system peak flow, game theory can be used in the allocation. In this method, both coincidence and non-coincidence peaks are taken into consideration [24, 25].

Among the five kinds of the allocation methods, the system peak state based method is the most frequently used method. It is reasonable for that the transmission network are planned and constructed mainly considering for the system peak condition.

\section{COST ALLOCATION OF UNUSED CAPACITIES}

There are always redundant capacities in the network, which is caused by the need of network security, the discrete investment of the transmission network, the uncertainty of demands and so on. The costs of the unused capacities should also be allocated in a rational way $[11,26]$.

\section{A. Postage method}

This method allocates the costs of unused capacities in proportion to the magnitude of the transmitted power, regardless of the location and influence of the generations and loads $[17,27]$. These are the simplest methods.

\section{B. Usage based allocation}

This method allocates the costs of unused capacities based on the usage of each user; that is, they allocate the costs of unused capacities according to the principle similar to that of the cost allocation of used capacities. For example, in [8], the costs of unused capacity of each branch are allocated among transactions in proportion to the absolute value of power flow on that branch caused by the transactions.

\section{Reliability based allocation}

This method allocates the costs of the unused capacities according to the users' reliability benefit from the power transmission or the impact of the users on system reliability and security $[10,11,25,28]$. For example, in [10], the costs of unused capacities of each branch are allocated in proportion to the reliability benefit of the transactions, which is defined as the increment of the probability of transaction failure caused by absence of the line. In [26], the total costs of the unused capacities of the network are allocated to native customers and wheeling customers according to their influence on system reliability and security, which is evaluated by EVV, the expected value of power flow variation in branches related to the transactions.

In the viewpoint of supporter of postage methods, the unused transmission capacity represents a common "system benefit" to all the users and therefore the costs should be paid for by all users uniformly [17]. In the viewpoint of supporter of usage based method, the network is constructed mainly for the need of actual transactions, and the redundant capacities are also caused by the basic demands. So all the costs should be allocated based on the usage. The reliability based methods considering another aspect, that is, system security or reliability.

\section{PRICING OF COUNTER FLOW}

The directions of power flows caused by different users may be different on the same branch. Usually, the flows having the same direction with the net flow is called positive flow, or dominant flow; the flows having the different direction with the net flow is called negative flow, or counter flow. Most power flow based methods have the problem of counter flow pricing $[7,8,26,29]$.

\section{A. Counter flows get credit}

This method allocates the costs among users in proportional to the algebraic values of the power flows caused by the users, which means that the price for the positive power flow is positive and that for the negative power flow is negative [18].

From the fact that counter flows benefit the network by 
lightening the line flow, counter flows should get credit. This method can provide the correct signal for the system operation, but they will not be easily accepted by some market participants since some of the transmission users who cause counter flow get credit instead of paying the charge.

\section{B. All flows pay according to absolute values}

This method allocates the costs according to the absolute values, instead of algebraic values, of power flows caused by users $[19,3031]$. That is, counter flows pay as if they are positive flows.

Ref. [32] argues that all flows should pay according to the absolute value. The counter flow and positive flow (dominant flow) are interdependent. Absent the dominant flow, the counter flow cannot exist. If the dominant flow disappears, the counter flow itself becomes the dominant flow. In [8], the costs of unused capacity are allocated using this method.

\section{Counter flow pay zero}

In this method, only positive flows pay for the transmission costs, counter flows neither pay money to nor get credit from the transmission owner. This method takes into consideration both the positive influence of counter flows and the acceptability. In [8], the costs of used capacity are allocated using this method.

How to charge for the counter flows is a disputable question. Different methods consider the question from different perspective, and every method is reasonable from its perspective. From the point of view of leading to economical network operation, the first method, that is, counter flows get credit method, is better.

\section{Pricing of REACtIVE POWER}

Since the transmission of reactive flows on the transmission network can reduce the capacity of active flow transmission, the influence of reactive flows must be considered in transmission fixed costs allocation. Therefore, firstly the total fixed costs should be allocated between branch active flows and reactive flows, and then the active flow cost and reactive flow costs should be allocated to related users respectively. The costs allocated to a transmission user will include 4 parts, that is, the active branch flow costs allocated to user active power and reactive power, and the reactive branch flow costs allocated to user active power and reactive power.

Since generators, branches and loads all can both generate and consume reactive power flow, it is difficult to decide which user the reactive power flows over branches come from and go to. So it is difficult to allocate the costs of reactive branch flows to users. In practice, most method neglect the influence of reactive flows and allocate all fixed costs to active power flows.

\section{ALLOCATION PRINCIPLES}

According to the allocation principles, transmission fixed costs allocation methods can be classified into corrected SRMC method, non-power-flow-based method, power flow based MWkm method, long-run incremental cost method, long-run marginal cost method and benefit based method.

\section{A. Corrected short-run marginal cost method}

In this method, the SRMCs are corrected to balance the transmission fixed costs. There are two kinds of corrections, that is, multiplicative correction and additive correction [1, $33,34]$.

Multiplication form:

$$
\rho_{k}(t)=\left[1+m_{k}(t)\right] \tilde{\rho}_{k}(t)
$$

Addition form:

$$
\rho_{k}(t)=\tilde{\rho}_{k}(t)+a_{k}(t)
$$

where $\bar{\rho}_{k}(t)$ is the price based on SRMC and $\rho_{k}(t)$ is the corrected price.

Since the transmission fixed costs is very large, the correction is significant and it may submerge the economic signal provided by SRMC.

\section{B. Non-power-flow-based methods}

The common characteristics of non-power-flow-based methods, which include the postage-stamp method, contract path method and distance based MWMile method, are that the actual influences of transactions are not considered in the allocation and it uses some predigested ways to determine the usage of the users to the transmission network [19].

In postage-stamp method, only the magnitude of transacted power is considered and neither the injection point nor withdrawal point is considered. This is the simplest method and it cannot provide any economic signal related with location.

In contract path method, it is assumed that power flows flow on the network according to the contracted path and the transmission fixed costs are allocated based on these paths. Since the paths of actual flows are always different with the contracted paths, this method may result in some degree of errors. This method is often used in the simplified calculation of transmission charges of wheeling transactions among different utilities.

Distance based MWMile method evaluates the usage of each user according to the product of the quantity of transacted power and the geographical distance between the source point and sink point. It is also a quite rough method. In practice, due to the effect of landform and branch types, there is no fixed relationship between the geographical distance and the actual costs.

All non-power-flow-based methods are simple and easy to calculate and apply, but since they do not take into consideration the effect of actual power flow and transmission users do not face their actual costs, it is not advantageous for the economic operation of the network.

\section{Power flow based $M W$-mile method}

Power flow based MW-mile method, which is firstly proposed by Shirmohammadi [6], takes into consideration 
both the quantity of transacted power and the electrical distance between source point and sink point, and allocates the total costs in proportion to the MW-mile of transactions.

$$
\begin{gathered}
C_{\text {total }, t}=C_{\text {total }} \frac{M_{t}}{\sum_{i \in T} M_{i}} \\
M_{t}=\sum_{k \in K} c_{k} L_{k} W_{t, k}
\end{gathered}
$$

where $C_{\text {toral }}$ is the total transmission fixed costs to be allocated, $C_{\text {total, } t}$ is the cost allocated to transaction $t, L_{k}$ is the length of branch $k, c_{k}$ is the cost of branch $k$ per mile per MW, $K$ is the set of all branches, $T$ is the set of all transactions, $W_{t, k}$ is the power flow in branch $k$ caused by transaction $t$.

Based on this method, many other new methods are proposed [29]. The common characteristic is that they all need to determine the influence or usage of each transaction on each branch. The main difference lies in how to determine the usage, that is, how to calculate the value of $W_{t, k}$.

\section{1) DC distribution factors based method}

The network Distribution Factors (DFs) traditionally used in power systems for security and contingency analysis can be casily adapted for the purpose of evaluate the transmission usage. There are three main kinds of DFs [2, 30]: GSDFs (Generation Shift DFs) [6], GGDFs (Generalized Generation DFs) [35 and GLDFs (Generalized Load DFs).

GSDFs, which represent the incremental use of node injections of the network, can be used to allocate the costs to node net injection. GGDFs, which represent the total use of the network of generations, can be used to allocate the costs to all generations. GLDFs, which represent the total use of the network of loads, can be used to allocate the costs to all loads.

All the three kinds of DFs are based on DC load flows. GSDFs are affected by the choice of reference nodes, but are not affected by the choice of operation conditions. On the other hand, GGDFs and GLDFs are not affected by the choice of reference nodes, but are affected by the choice of operation conditions.

\section{2) Power flow tracing based method}

In 1996, Bialek and Kirschen almost simultaneously proposed a kind of power flow tracing method independently. The basic of both the two tracing methods is the proportional sharing principle. In Bialek method, it is assumed that the nodal inflows are shared proportionally among the nodal outflows [16, 17, 36]. In Kirschen method, it is assumed that for a given common (a set of contiguous buses supplied by the same set of generators), the proportion of the inflow traced to a particular generator is equal to the proportion of the outflow traced to the same generator $[23,37,38]$.

Both methods can answer the question of how much power flow on a branch coming from a particular generator and consumed by a particular load. The topological distribution factors are always positive, thus eliminating many problems resulting from counter flows. On the other hand, all-positive characteristic reflect a disadvantage of this method, that is, it cannot provide enough economic information.

There are many improved or modified power flow tracing methods. The main differences of these methods mainly lie with the way to treat losses, [39], the way to treat reactive power[40, 41], the way to treat loop flows [42] and the arithmetics $[43,44]$.

\section{3) Power flow comparison method}

In power flow comparison method, the impacts of the users on branch flows are evaluated by comparing power flows under different conditions.

(1) Marginal comparison method

In marginal comparison method, the usage of each user on each branch flow is evaluated based on the marginal flow of the user, which is the difference between branch flows of the base case (without any transactions) and the case with only the studied user.

$$
F_{M, i}=F_{\mathrm{i}}-F_{0}
$$

where $F_{M, i}$ is the marginal flow caused by user $i, F_{\mathrm{j}}$ is the branch flow when only user $i$ exists, $F_{0}$ is the branch flow of the base case. In different conditions, the base case can have different definitions. For instance, when the costs to be allocated are the incremental costs caused by the wheeling transactions, the based case is usually defined as the case when all the wheeling transactions do not exist but the native customers exist; when all transmission users are treated equally and the allocated costs are the total costs, the base case is the zero case which means no any user exists. Thus, the marginal flow of a user would be the flow when only the studied transaction exists. Using DC load flow model, the marginal flow can be calculated using GSDF method.

(2) Incremental comparison method

In incremental comparison method, the usage of each user on each branch flows is evaluated based on the incremental flow of the user $[8,18]$, which is defined as the difference between branch flows of the operation case (with all transactions) and the case with all transactions except the studied user, that is,

$$
F_{l, i}=F_{T}-F_{T, i}
$$

where $F_{l, i}$ is the incremental flow caused by transaction $i, F_{T, i}$ is the branch flow with all transactions except transaction $i$, $F_{T}$ is the branch flow of the operation case. Using DC load flow model, for bilateral transactions, the incremental flows are the same with the marginal flows [8].

(3) The aggregated comparison method

In the aggregated comparison method, the usage of each user on each branch is evaluated based on the aggregated flow of the user, which is defined as the weighted sum of the corresponding marginal flow and incremental flow [17].

$$
F_{A, i}=a^{*} F_{M, i}+b * F_{l, i}
$$

where $F_{A, i}$ is the aggregated flow of transaction $i$. Usually, the aggregated flow is the average value of the corresponding marginal flow and incremental flow, that is, $a=b=0.5$ [17].

In marginal comparison method, it is regarded that the studied user is the first user added to the network. In incremental comparison method, it is regarded that the studied 
user is the last one added to he network. In the aggregated comparison method, the impact of the studied user on the branch flow as both the first user and the last user are considered.

\section{4) Current based method}

In this method, the power injections are converted into current injections, and the impacts of current injections on branch flows are evaluated. The current based methods can be further compartmentalized into two categories, that is, current conjugate based method and cross term based method.

In current conjugate based method, node voltages are considered to be constants or to be caused jointly by all the users, and the costs are allocated to users in proportion to the conjugate of branch currents caused by them $[45,46]$. The allocation formula can be written as

$$
\begin{gathered}
S_{i j}=V_{i} I_{i j}^{*}=V_{i} \sum_{t \in T} I_{i j, t}^{*}=\sum_{t \in T} V_{i} I_{i j, t}^{*}=\sum_{t \in T} S_{i j, t} \\
S_{i j, t}=V_{i} I_{i j, t}^{*}
\end{gathered}
$$

where $S_{i j}$ and $I_{i j}$ are the complex power and current on branch ij, $\quad V_{i}$ is the complex voltage at node $i, I_{i j, r}$ is the complex current on branch $i j$ caused by user $t, T$ is the set of all user, $S_{i j, t}$ is the complex power flow on branch $i j$ caused by user $t$. $I_{i j, l}$ can be calculated through different ways. Since the currents conform to superposition principles, if each current injection is regarded as a current source, according to circuit theory, it is easy to get the contribution of each current source to the branch currents.

In cross term based method, the node voltage is expressed as the function of user currents, so the branch flow is also a function of user currents $[9,31]$. The branch flow functions are nonlinear functions, in which include both independent terms, each of which relates to only one user current, and cross terms, each of which relates to at least two user currents In cross term based method, each independent term is allocated to the respective user and each cross term is allocated to the corresponding multiple users based on a certain principle, such as average allocation, allocation in proportion to their current injections and so on. The allocation formula can be written as:

$$
\begin{gathered}
S_{i j}=\sum_{t \in T} f_{t}\left(I_{t}\right)+S_{i j, \text { cross } s} \\
S_{i j, t}=f_{t}\left(I_{t}\right)+S_{i j, \text { cross }, t}, \sum_{t \in T} S_{i j, \text { cross }, t}=S_{i j, \text { cross }}
\end{gathered}
$$

where $f_{t}\left(I_{t}\right)$, a function of $I_{t}$, is the independent term caused by user $t, S_{i j, c r o s s}$ is the sum of cross terms, $S_{i j, c r o s s, t}$ is the cross terms allocated to user $t$.

\section{5) Sensitivity methods}

Sensitivity methods evaluate the effect of users on branch flows based on the sensitivity of branch flows to the quantities of the transmitted power $[19,47]$.

$$
W_{t, k}=u_{t}^{k} q_{i}, \quad u_{i}^{k}=\partial f_{k} / \partial q_{t}
$$

where $u_{i}^{k}$ is the sensitivity of power flow on branch $k$ to the magnitude of user $t, q_{t}$ is the magnitude of user $t, f_{k}$ is the power flow on branch $k$.

If DC load flow is used the sensitivities of the node injections are GSDFs. The summation of the contribution of all users to a branch flow calculated by sensitivity method is not always equal to the total power flow on the branch. An improved method is to consider sensitivities at multiple states to get more reasonable result $[13,48]$.

\section{Benefit based method}

In this method, the costs are allocated according to the benefits obtained by users though the network usage, not the impact of users on the network [27, 49-52].

The benefit of transmission network usage mainly stem from three aspects [49]: the reduced overall cost of generation; the reduction or elimination of unserved demands in generation deficient zones or portions of the network; the increased quality and security of supply. Evidently, the benefit of loads may be quantified in terms of the reduction of unserved demands, the reduction of electricity price and the improvement of electricity quantity, and the benefit of generators may be quantified in terms of the increment of profits from the increment of generation quantity and the reduction of generation costs $[27,51]$. In [49] the demand function is deduced according to the benefit function, and then the price of each branch is calculated based on the maximum benefit of the branch.

\section{E. Long-run incremental cost method}

Long-run Incremental Cost (LRIC) methods can be used to allocate the incremental transmission fixed costs to the wheeling transactions causing them. There are two main LRIC methods, that is, the standard LRIC method and the long-run fully incremental method [5].

In the standard LRIC method, the traditional system planning approaches are used to determine the required reinforcements with and without wheeling transactions, and the difference between the costs of the two cases is regarded as the costs caused by the wheeling transactions. If more than one wheeling transactions present in the studied period, the total incremental costs have to be allocated to each wheeling transaction.

In the long-run fully incremental method, the excess transmission capacities are not allowed to be used by wheeling transactions and the wheeling transactions are forced to make reinforcement along the path of the wheeling transactions to accommodate them. The system planning approaches are not utilized in this method, but the required reinforcement is estimated. If more than one transaction exist, it is needed to calculate the reinforcement required by each transaction. Since each transaction is already separately considered, the cost does not have to be allocated among the separate transactions.

\section{F. Long-run marginal cost method}

Long-run Marginal Cost (LRMC) method allocates transmission fixed costs based on the LRMC of transmission services $[15,22,53]$. The LRMC of a transmission 
transaction is the sensitivity of the network capacity cost to the quantity of the transmitted power, that is, the least network reinforcement costs needed by per unit increment of power transmission.

LRMC are different with LRIC in that LRMC represents the incremental cost, which is the additional cost caused by a whole transaction, while LRMC represents the impact of an infinitely small change of the transaction on the cost.

Since the accurate calculation of LRMC is very difficult, some simplifications are often taken. The most often used simplifications include [14, 22]:

a) the capacity of transmission branches can be increased continuously;

b) there are no new rights of ways;

c) the peak demand condition is considered;

d) all the lines are of the same type;

e) the costs of branches are linear functions of branch capacities.

Under these simplifications, LRMC is the sensitivity of system MW-Mile to the quantity of the transmitted power [16].

There are two kinds of LRMC methods, that is, Investment Cost-Related Pricing (ICRP) method and DC load flow pricing (DCKFP) method.

In ICRP method [15], it is assumed that electric power can be routed at will on the existing routes. Kirchhoff's voltage law is ignored, and the shorter routers are being used. The mathematical model can be written as:

$$
\begin{gathered}
\min v=\left(\sum_{i j}\left|l_{i j} f_{i j}\right|\right) \\
\text { S.t. } \quad \sum_{j} f_{i j}=P_{i}, \text { for every bus } i \\
\lambda_{i}=\frac{\partial v^{*}}{\partial P_{i}}, \quad L R M C_{i}=c \lambda_{i}
\end{gathered}
$$

where $l_{i j}$ is the length of branch $i j, f_{i j}$ is the power flow on branch $i j, c$ is the expansion constant (reflect the cost of transmission expansion per mile per MW).

In DCLFP method [14], DC power flow simplifications are assumed. The network satisfies Kirchhoff's laws. Replacing $f_{i j}$ in the above formula with $b_{i j} \theta_{i j}$, we can get the mathematical model of DCLFP method. $b_{i j}$ is the reactance of branch $i j, \theta_{i j}$ is the difference between the voltage angles of the two terminal of branch $i j$.

\section{CONClusions}

The transmission fixed costs allocation method is an important aspect of electricity market design. A reasonable allocation method could lead to efficient utilizations of existing transmission facilities and, at the same time, provide economic signals for guiding future generation planning and load sitting

This paper makes overall, systematic summary and analysis of the existing allocation methods which are classified according to seven different criteria. Due to the difference in the background, the viewing angle, the understanding of the function of the transmission network and the main allocation goal, different methods have different emphasis and adopt different simplifications. In the designing of transmission fixed costs allocation method, the real situation which includes the political and economical background, the network condition, the market model and so on must be taken into consideration. Neither existing method can be imitated rigidly.

\section{RFFERENCES}

[1] F.C. Schweppe, M.C. Caramanis and R.D. Tabors, Spot Pricing of Electricity, Boston: Kluwer Acadenic Publishers. 1988.

[2] H. Rudnick, M. Soto and R. Palma, "Use of system approaches for transmission open access pricing," Electrical Power and Energy Systems, vol. 21, no. 2, pp.125-135, 1999.

[3] D.Shirmohammadi. X.V.Filho.B and Gorenstin et al, "Some fundamental technical concepts about cost based transmission pricing." IEEE Transactions on Power Systems, vol. 11. no. 2, pp.1002-1008, May 1996.

[4] Federal Energy Regulatory Commission (FERC). Working Paper on Standardized Transmission Service and Wholesale Electric Market Design, http://www.ferc.org.

[5] H.H. Happ, "Cost of wheeling methodologies," IEEE Transactions on Power Systems, vol. 9. no. 1, pp.147-156, Feb. 1994.

[6] D. Shirmohammadi, P.R. Gribik and E.T.K. Law et al, "Evaluation of transmission network capacity use for wheeling transactions," IEEE Transactions on Power Systems, vol. 4, no. 4, pp.1405-1413, Oct. 1989.

[7] R.R.Kovacs and A.L.Leverett, "A load flow based method for calculating embedded. incremental and marginal cost of transmission capacity." IEEE Transactions on Power System.s, vol. 9, no. 1, pp.272-278, Feb. 1994.

[8] J.W.Mlima , M.V.F.Pereira and Pereira J L R, "An integrated framework for cost allocation in a multi-owned transmission system," IEEE Transactions on Power Systems, vol. 10, no. 2, pp.971-977, may 1995.

[9] A. Zobian and M. Ilic, "Unbundling of transmission and ancillary services Part I: Technical issuesPart I: Technical issues." IEEE Transactions on Power Systems, vol. 12, no. 2, pp.539-548, Jan. 1997.

[10] C.W. Yu and A.K. David, "Pricing transmission services in the context of industry deregulation," IEEE Transactions on Power Systems, vol. 12, no. 2, pp.503-510, Feb. 1997.

[11] E.L. Silva, S.E.C. Mesa and M. Morozowski, "Transmission access pricing to wheeling transactions: a reliability based approach," IEEE Transactions on Power Systems, vol. 13, no. 4, pp.1481-1486, Nov. 1998.

[12] M.E. Baran, V. Banunarayanan and K.E. Garren, "A transaction assessment method for allocation of transmission services," IEEE Transactions on Power Systems, vol. 14, no. 3, pp.920-928, March 1999.

[13] A. Fradi, S.Brignone and B.F.Wollenber, "Calculation of Energy Transaction Allocation Factors," IEEE Transactions on Power Systems. vol. 16, no. 2, pp.266-272, May 2001.

[14] C.W.Yu, "Long-run marginal cost based pricing of interconnected system wheeling," Electric Power Systems Research, vol. 50, no. 3, pp.205-212, 1999.

[15] M.C. Calviou. R.M. Dunnett, and P.H. Plumptre, "Charging for use of a transmission system by marginal cost methods," Proc. Eleventh Power Systems Computation Conference, Avignon, France, August 30 September 4 1993, pp.385-39l, Aug. 1993.

[16] J.Bialek, "Topological generation and load distribution factors for supplement charge allocation in transmission open access," IEEE Transactions on Power Systems, vol. 12, no. 3, pp.1185-1193, Aug. 1997.

[17] J.Bialek, "Allocation of transmission supplementary charge to real and reactive loads," IEEE Transactions on Power systems, vol. 13, no. 3 , pp.749-754, August 1998.

[18] J.Yang and M.D.Anderson, "Tracing the flow of power in transmission networks for use-of-transmission-system charges and congestion management," IEEE Power Engineering Society 1999 Winter Meeting. New York, NY, USA, vol. 1, pp.399-405, 1998.

[19] Y.-M. Park, J.-B. Park and J.-U. Lim et al, "An analytical approach for transaction costs allocation in transmission system," IEEE Transactions on Power Systems, vol. 13, no. 4, pp.1407-1412, Nov. 1998.

[20] R.J.Camfield and A.G.Schuster, "Pricing transmission services efficiently," The Electricity Journal. vol. 13, no. 9. pp.13-32, Nov. 2000. 
[21] R.D.Tabors, "Transmission system management and pricing: new paradigms and international comparisons," IEEE Transactions on Power Systems, vol. 9. no. 1, pp.206-215. Feb. 1994.

[22] A. Bakirtzis. P.Biskas and A.Maissis et al, "Comparison of two methods for long-run marginal cost-based transmission use-of-system pricing." IEE Proceedings-Generation, Transmission and Distribution, vol. 148, no. 4, pp.477-488. July 2001.

[23] D. Kirschen. R. Allan and S. Ahrned, "Allocating transmission system usage on the basis of traceable contributions of generators and loads to flows," IEEE Transactions on Power Systems, vol. 13, no. 2, pp.527-523. May 1998.

[24] Y. Tsukamoto and I. Iyoda, "Allocation of fixed transmission cost to wheeling transactions by cooperative game theory," IEEE Transactions on Power Systems, vol. 11, no. 2, pp.620-629, May 1996.

[25] C.W. Yu and A.K. David. "Integrated approach to transmission services pricing," IEE Proc.-Gener. Transm.Distrib., vol. 146, no. 3, pp.255-260. May 1999.

[26] P.Jiuping. Y.Teklu and S.Rahman et al, "Review of usage-based transmission cost allocation methods under open access," IEEE Transactions on Power Systems, vol. 15, no. 4, pp.1218-1224, Nov. 2000.

[27] I.J. Perez-Arriaga, F.J. Rubio and J.F. Puerta et al, "Marginal pricing of transmission services: an analysis of cost recovery," IEEE Transactions on Power Systems, vol. 10, no. 1, pp.546-553, Feb. 1995.

[28] N. Gu, X. Ling and Z. Hou, " A New approach for pricing of fixed costs in wheeling rates by modified Mile-Power method," Power System Technology, vol. 23, no. 2, pp.66-69, Feb. 1999

[29] J.W.M.Lima, "Allocation of transmission fixed charges: an overview," IEEE Transactions on Power Systems, vol. 11, no. 3, pp.1409-1418. Aug. 1996.

[30] H.Rudnick, R. Palma and J.E.Fernandez, "Marginal pricing and supplement cost allocation in transmission open access," IEEE Transactions on Power System., vol. 10, no. 2, pp.1125-1142, May 1995.

[31] A.Zobian and M. Ilic, "Unbundling of transmission and ancillary services Part II: Cost-based pricing frameworkPart I: Technical issues," IEEE Transactions on Power Systems, vol. 12, no. 2, pp.539-548, Jan. 1997.

[32] G. Gross and S. Tao, "A physical-flow-based approach to allocating transmission losses in a transaction framework," IEEE Transactions on Power Systems, vol. 15, no. 2, pp.631-637, May 2000.

[33] M.C.Caramanis, N.Roukos and F.C.Schweppe, "WRATES: a tool for evaluating the marginal cost of wheeling," IEEE Transactions on Power Systems, vol. 4, no. 2, pp.594-605, May 1989.

[34] H.M.Merrill and B.W.Erickson. "Wheeling rates based on marginal-cost theory," IEEE Transactions on Power Systems, vol, 4, no. 4, pp.14451451. Nov. 1989

[35] W.Y.Ng, "Generalized generation distribution factors for power system security evaluation," IEEE Transactions on Power Apparatus and Systems, vol. 100, no. 3, pp.1001-1005, March 1981.

[36] J.Bialek, "Tracing the flow of electricity," IEE Proc.-Gener. Transm.Distrib., vol. 143, no. 4, pp.313-321. July 1996.

[37] D. Kirschen, R. Allan and G. Strbac, "Contributions of individual generators to loads and flows," IEEE Transactions on Power Systems, vol. 12, no. 1, pp.52-60. Feb. 1997

[38] D. Kirschen and G. Strbac, "Tracing active and reactive power between generators and loads using real and imaginary currents," IEEE Trunsactions on Power Systems, vol. 14, no. 4, pp.1312-1319, Nev. 1999.

[39] Z. Li, M Pan, S. Yang and X. Chen, "Study on tracing power flow," Electricity Power, vol. 33, no. 11, pp.41-43, Nov. 2000.

[40] B. Chang, H. Sun and J. Zhou, "Theory and approach to power flow tracing)," Power System Technology, vol. 22, no. 1, pp.68-71, Jan. 1998.

[41] Y. Dai, Y. Ni and F. Wen et al, "Reactive power pricing based on cost allocation through power flow tracing," Automation of Electric Power Systems, vol. 24, no. 18, pp.13-17, 92000

[42] P. Wei, Y. Ni and F. F. Wu, "Load flow tracing in power systems with circulating power," Electrical Power and Energy Systems, vol. 15, no. 3, pp.807-813, 2002.

[43] F.F. Wu, Y.X. Ni and P. Wei, "Power transfer allocation for open access using graph theory-fundamentals and applications in systems without loopflow," IEEE Transactions on Power Systems, vol. 15, no. 3, pp.923929, Aug. 2000.

[44] F.Cubina, D. Grgic and I.Baniĉ. "A method for determining the generators' share in a consumer load," IEEE Transactions on Power Systems, vol. 15. no. 4. pp. 1376-1381, Nov. 2000.
[45] Qiao Zhenyu, Chen Xueyun and Zhang Lizi et al, "A new concept of the separation of electrical power flow," Proceeding of the CSEE, vol. 21, no. 1, pp.77-79, Jan. 2001.

[46] Qiao Zhenyu. Chen Xueyun and Zhang Lizi et al, "Cost allocation of transmission line in power market)," Automation of Electric Power Systems, vol, 26, no. 12, pp.19-23.62002.

[47] J.G.Vlachogiannis, "Accurate model for contribution of generation to transmission system effect on charges and congestion management," IEE Proceedings-Generation, Transmission and Distribution, vol. 147, no. 6. pp.342-348. Nov. 2000

[48] Z. Wu, "Contribution factor theory on variables to the nonlinear function value and its applications." Automation of Electric Power Systems, vol. 24, no. 22. pp.32-34, Nov. 2000

[49] E.D.Farmer, B.J. Cory and B.L.P.P. Perera, "Optimal pricing of transmission and distribution services in electricity supply," IEE Proceedings-Generation, Transmission and Distribution, vol. 142, no. 1 , pp.1-8, Jan. 1995

[50] E.D.Farmer, B.L.P.P. Perera and B.J. Cory, "Optimal pricing of transmission services: application to large power systems," IEE Proc.Gener. Transm. Distrib., vol. 142, no. 3, pp.263-268. May 1995.

[51] F.J. Rubio and I.J. Perez-Arriaga, "Marginal pricing of transmission services: a comparative analysis of network cost allocation methods," IEEE Transactions on Power Systems, vol. 15, no. 1, pp.448-454, Feb. 2000.

[52] P.D.C.Wijayatunga, B.J.Cory and M.J.Short, "Security and revenue reconciliation in optimal transmission pricing." IEE Proc.-Gener Tran.m.Distrib., vol. 146, no. 4, pp.355-359, July 1999.

[53] R.Green, "Transmission pricing in England and Wales," Utility Policy, vol. 6. no. 3. pp.185-193. 1997

\section{BIOGRAPHIES}

Zhaoxia Jing was born in Henan, China, in 1975. She received her B.E. degree in electrical engineering from the Huazhong University of Science and Technology(HUST) in 1997 and now she is pursuing her Ph.D. in the same university. Her major area of work is electricity market.

Xianzhong Duan was born in Hunan, China, in 1968. He graduated from HUST in 1987, and received his Ph.D. degree in electrical engineering at the same university in 1992. Since then he has been working at HUST, now he is a full profestor. His research interests include power system analysis, electricity market and informational of power systems.

Fushuan Wen received his BE and ME degrees from Tianjin University, China, in 1985 and 1988, respectively, and PhD from Zhejiang University, China, in 1991, all in electrical engineering. He joined the faculty of Zhejiang Unversity, China, in 1991, and has been professor there since 1997. He held a visiting position in the National University of Singapore from 1995 to 1997, and was a research fellow in the Hong Kong Polytechnic University from July 1998 to February 2001. He is now a research assistant professor in the University of Hong Kong. His current research interests are in power industry restructuring and artificial intelligence applications in power systems.

Yixin Ni (Senior Member, IEEE) received her B. Eng., M. Eng., and Dr. Eng. all from Electrical Engineering Department, Tsinghua University, P.R. China in 1968, 1981 and 1983 respectively. Her research interests are in power system modeling, simulation, stability and control, and power electronics applications in power systems. She was a professor of Tsinghua University and is now with the University of Hong Kong. She is a senior member of CSEE and Fellow of HKIE.

Felix F. Wu (Fellow, IEEE) received his Ph. D. from University of California at Berkeley (UCB). He is now a chair professor of electrical engineering at the University of Hong Kong. Prior to that he was a professor and vice chairman of Dept. of EECS, UCB. His research interests are in power industry restructuring. power system investment planning, design of modern control centers, distribution automation, distributed processing etc. 